\title{
Kinematics Analysis of Kicking during Swing of Soccer Players
}

\author{
Fuming $\mathrm{Gu}^{1, \mathrm{a}}$ \\ ${ }^{1}$ Department of Sports Medicine, Chengdu Sport University, Chengdu 610041, China; \\ a1165037369@qq.com
}

Keywords: Kinematics, soccer player, kicker, biomechanics

\begin{abstract}
The skill of swing period of kicking leg is an important factor to affect the athletic performance of soccer player. This paper analyzed the swing period using the biomechaical methods. The findings indicated that (1) the swinging process can be divided into forceful stretching, active backward swinging, forward thigh swinging, forward leg swinging stage, and kicking ball stage according to the method of combining the swinging sequence of the swinging leg with the swinging speed in soccer playing; (2) forceful stretching stage is an important part that cannot be ignored; (3) at the active backward swinging stage, the swinging leg should swing as forceful as possible under the precondition that it does not affect the forward swinging speed; (4) after the thigh swing backward to the most extent, it should get into the forward thigh swinging stage quickly in order to avoid loosening anterior muscles of the thigh and destroying the integrity of plyometric contraction; and (5) the range of forward leg swinging should not be too large.
\end{abstract}

\section{Introduction}

Some scholars performed a fewer researches on kicking of soccer players. Zago et al. put the focus on anatomical angles, contribution of upper limbs in kick biomechanics, kinematics of the body center of mass, which describes the whole body movement and is related to balance and stability when kicking ball. It has concluded that differences in motor control between preferred and non-preferred leg kicks exist, particularly in the movement velocity and upper body kinematics.[1] Arundale et al. reviewed the existing literature elucidating the biomechanics of kicking a soccer ball and propose a progressive kicking program to support clinicians in safely returning their soccer athletes to the demands of sport.[2] Kapidžić et al. established which applied kinematic variables significantly contributed to the efficiency of the instep kick motion in soccer. The finding revealed that two variables significantly contributed to the speed of the ball: speed of the foot of the kicking leg at the time of contact with the ball and the distance between the angle support leg and center of the ball.[3] Bjelica attempted to gain relevant knowledge about important differences with respect to compare accuracy of instep kicking between preferred and non-preferred leg depending on the different intensity in a resting state, and in a state of fatigue. The findings clearly demonstrated that the young football players have much more uniformed legs at the expense of accuracy when fatigued and kick the ball with the maximal intensity.[4] Marques et al. compared the ball velocity in different kicking conditions with dominant and non-dominant leg in junior soccer players. The study showed that kicking velocity was influenced very much under different conditions when kicking with the dominant leg while for the non-dominant leg this influence was small.[5] Dörge et al. examined the release speed of the ball in maximal instep kicking with the preferred and the non-preferred leg and to relate ball speed to biomechanical differences observed during the kicking action. The finding conclude that the difference in maximal ball speed between the preferred and the non-preferred leg was caused by a better inter-segmental motion pattern and a transfer of velocity from the foot to the ball when kicking with the preferred leg.[6] Cerrah et al. defined activation characteristics of knee muscles of the kicking leg during the in-step kick and interpret these in relation to isokinetic strength parameters and ball velocity in professional compared to amateur soccer players. The study indicated that the superior performance of professional players compared to amateurs appears not to be due to muscle strength factors but rather to subtle differences in technique, as indicated by EMG data, throughout both the build up and execution of 
the kick.[7] Scurr et al. examined EMG activation in three quadriceps muscles during a kicking accuracy task. The outcome indicated that no significant difference were observed between muscles or kick phases, kicks to the right targets produced significantly greater muscle activity than those towards the left targets.[8] Scurr \& Hall examined the effects of approach angle on kicking accuracy and three-dimensional kinematics of penalty kicks. The study has concluded that altering an individual's self-selected approach angle at recreational level did not improve kicking accuracy or ball velocity, despite altering aspects of underlying technique.[9] Based on the literature review, the author will discussed the technique of kicking using kinematics.

\section{Phase division of ball kicking leg of the swinging stage}

Phase division is an important part to research the skilled movements. The division bases of movement phase are always different due to different research objects, purposes, and focuses. Generally, they must objectively show both the actual movement structure and the actual measurement. According to the sequence and speed of the swinging leg, the swinging process can be divided into 5 stages: 1) Forceful stretching stage: forceful stretching stage starts from "the kicking leg is on the ground" to " the leg pushes off the ground to kick the football". For the kicking leg stretching forcefully and having not left the ground, kicker's thigh will not move forward with hip joint in parallel , and the thigh can only be supported by kicking leg and rotate backward relatively by pivoting on the hip joint. At the same time, the range of extension of the thigh is increasing gradually relative to that of the hip joint. In this phase, the swinging of kicking leg is mainly finished by pushing off the ground and stretching the hip. 2) Active backward swinging stage: active backward swinging stage starts from the "the kicking leg pushing off the ground" to the "supporting foot standing on the ground". After pushing off the ground, the kicking leg starts to swing. In this stage, as the thigh of kicking leg has been swinging backward extremely, with the crus bending at the same time, which leads to "passive insufficiency" of some double joint muscles such as rectus femoris and sartorius . For the crus, it causes "active insufficiency" of some hamstring muscles such as semi-tendinosus and semi-membranosus. Therefore, in order to overcome the "passive insufficiency" and "active insufficiency", and raise the backward swing scope of kicking leg, the kicker must exert his strength actively. 3) Forward thigh swinging stage: Forward thigh swing stage starts from the "supporting leg standing on the ground" to "the knee joint of kicking leg reaches the maximum speed". At this moment, the backward swinging stage is ending, and the kicking leg starts to swing forward. Hip joint accelerates first prior to ankle joint, and then the knee joint speeds up, which conforms to the principle of sequential joint movements. The angle of thigh (the angle between $\mathrm{X}$ axis in negative direction and hip-knee attachment) gradually decreases. As the knee swings forward, the phenomenon of "active insufficiency" and "passive insufficiency" disappears. The speed of leg curvature movement increases a lot, which increases the backward swinging amplitude of kicking leg, and provides favorable conditions for the acceleration of the forward swing. 4) Forward leg swinging stage:forward leg swinging stage starts from "the knee joint of kicking leg reaches the maximum speed" to "the moment kicking the ball". In this phase, the speed of knee joint will fall sharply after it reaches the peak, while the speed of ankle joint begins to fall quickly, with the momentum transferred from the thigh to the leg gradually. 5) Ball kicking stage: this stage starts from "kicking the ball" until "the ball leaves away from the upper surface of foot". Studies have shown that, in addition to main factors such as thigh and leg, the hardness of foot and sneakers and tiny changes in contact with the ball are also important reasons to influence the speed and accuracy. The player who can kick the ball of high speed, usually has a strong buckled state of ankle. 


\section{Analysis of the swinging leg at different stages.}

This study mainly analyzes the skills at 4 stages, including forceful stretching, active backward swinging, and forward thigh swinging and forward leg swinging stage.

1) Forceful stretching stage

It is a process of brake buffer, from the kicking foot touching the ground to entire sole of the foot on the ground. Meanwhile it forms an axis of rotation pivoting on the hip joint and rotating backward relative to the body, so the angular velocity of thigh is high, with a value of $196.85 \pm 23.45)^{\circ} / \mathrm{s}$. After the buffer stage, the thigh will be stretching upward for a short time. During this period, the angle velocity will decrease, but it will increase higher and higher when getting into stretching stage due to forceful stretching forward of the kicking leg. However, it will decrease gradually with the sole lifting from the ground. Earlier in this stage, the sole will push off the ground gradually, so the angular velocity of leg will increase first and then decrease. However, the angular velocity starts to increase later in this stage, because the sole will push off the ground slowly and the hamstring muscle group and the triceps surae muscle also begin to participate in work to make the thigh actively stretching backward. From the point of joint angle, as a result of forceful stretching of the swinging leg, both thigh angle and knee angle will tend to increase all the time, with the maximum angle of $101.8 \pm 76.5^{\circ}$ and $163.7 \pm 7.8^{\circ}$ respectively, while the leg angle (the angle between $\mathrm{x}$ axis in negative direction and ankle-knee attachment) will always tend to decrease. At this stage, the higher the stretching efficiency is, the bigger initial momentum of forward swinging will the swinging leg get. As a result, to improve the stretching efficiency of the swinging leg is one of the important ways to raise the swinging speed of the swinging leg at the forceful stretching stage.

2) Active backward swinging stage

Back swing phase is mainly on the basis of the thigh stretching forcefully to a certain extent, thus the backward swinging angular velocity of the thigh will gradually decrease at last, until up to the limitation of backward swinging, and with angular velocity close to 0 . Finally, the maximum swing amplitude is up to $114.2 \pm 9.8^{\circ}$. Compared with the previous stage, the thigh angle only increased by $6.60^{\circ}$. From the point of leg angular velocity, it will increase sharply, which indicates that the leg has begun to accelerate in the process of curvature movement, and the leg angular velocity reaches the maximum value of $558.4 \pm 46.1 \%$ s. Why the leg angular velocity is so high? This is because in the previous stage, the excellent stretching of the kicking leg creates a favorable condition to make the thigh and leg stretching straight completely, which stretches the triceps surae, hamstrings and other major muscle groups, forming a better plyometric stretching shape, so the leg can accelerate to move toward the thigh. Therefore, the backward swinging of the thigh is mainly finished at the forceful stretching stage, while the active backward swinging is immediately following the forceful stretching stage. After that, the leg continues to speed up for backward swinging, until the swing speed up to the maximum value.

3) Forward thigh swinging stage

With end of backward swinging, the swinging thigh starts to swing forward, in this phase the forward swinging angular velocity of thigh shows a trend of "increase-decrease-increase" and the backward swinging angular velocity of leg decreases gradually, until the leg stops swinging backward and starts to accelerate to swing forward. Hip joint shows a downward trend in speed after it reaches the maximum value, which is a braking process, and the result of the braking is the completion of momentum transfer from the near-end to the far-end, making the thigh angular velocity increase to $161.8 \pm 7.6 \%$ and knee joint's resultant velocity reach $5.6 \mathrm{~m} / \mathrm{s}$; At the same time, active braking of hip also leave more time and space for the thigh and leg to swing forward, because the body has a certain swing amplitude, if the hip joint doesn't brake in time, the swing amplitude of thigh and leg will be decrease accordingly, and finally the resultant velocity of foot will decrease significantly; From the point of anatomy, the active braking of hip joint can make the muscles on it more firmly, which is more favorable to the contraction of thigh muscles, so that an ideal muscle strength will be formed. In addition, the leg begins to accelerate to swing forward after 
its angular velocity is reduced from the maximum to 0 . At this moment, the leg has the largest buckling degree and gets close to the thigh to the most extent, with the completion of transfer from backward curvature movement to forward stretching movement.

4) Forward leg swinging stage

After knee joint reaches maximum velocity, it begins to enter the forward leg swinging stage, and the speed of ankle joint will reach a peak value. The gradual deceleration of knee joint is a braking process, which makes the momentum transferred from the near-end to far-end: from the thigh to the leg, causing the angular velocity of leg increases dramatically, and reaches the maximum value of $802.7 \pm 62.5 \%$ s in the whole swinging stage. However, it is found that the leg swinging angular velocity is reduced just when the kicking leg touches the ball, with the value of $634.7 \pm 31.6^{\circ} / \mathrm{s}$. This may be associated with the experimental design of experiments. In order to control the kicking accuracy, the kicker will control the speed actively, which is consistent with the actual game, because in the game, no matter passing or shooting, the player will not only pursuing speed, but the accuracy should be the most important. In addition, the thigh is still swinging forward at the forward leg swinging stage. Therefore, the braking of thigh is not absolute, and its angular momentum is not completely transmitted to the leg, so the process of leg gaining momentum includes 2 parts. That is: Part of the near-end transmission and the other part of far-end application of force. In addition, the knee joint angle of the swinging leg is about $136.5 \pm 9.4^{\circ}$ when kicking the ball, which is basically consistent with the predecessors' research results.

\section{Conclusion}

The swinging process can be divided into 5 movement phases according to the method of combining the swinging sequence of the swinging leg with the swinging speed in soccer playing.

Forceful stretching stage is an important part that cannot be ignored. At the final step of approach, the player must try to stretch the kicking leg fully and quickly.

At the active backward swinging stage, the swinging leg should swing as forceful as possible under the precondition that it does not affect the forward swinging speed. After the thigh swing backward to the most extent, it should get into the forward thigh swinging stage quickly in order to avoid loosening anterior muscles of the thigh and destroying the integrity of the super-equal-length contraction; the range of forward leg swinging should not be too large.

The moving characteristics of 5 movement phases of the swinging leg are different from each other, which requires both improving the accuracy and speed of passing and shooting, and must form a correct dynamic stereotype of movement technology.

\section{Acknowledgments}

This study was supported by the Sports Medicine key laboratory of General Administration of Sport of China/Sports Medicine key laboratory of Sichuan province Foundation (No:2015CTYY003) .

\section{References}

[1] Zago, Matteo; Motta, Andrea Francesco; Mapelli, Andrea; Annoni, Isabella; Galvani, Christel; Sforza, Chiarella, Effect of Leg Dominance on The Center-of-Mass Kinematics During an Inside-of-the-Foot Kick in Amateur Soccer Players. Journal of Human Kinetics Oct 2014, Vol. 42 Issue 1, p51

[2] Arundale, Amelia; Silvers, Holly; Logerstedt, David; Rojas, Jaime; Snyder-Mackler, Lynn, An Interval Kicking Progression for Return to Soccer Following Lower Extremity Injury. International Journal of Sports Physical Therapy Feb2015, Vol. 10 Issue 1, p114

[3] Kapidžić, Alen; Huremović, Tarik; Biberovic, Alija, Kinematic Analysis of the Instep Kick in Youth Soccer Players. Journal of Human Kinetics Oct2014, Vol. 42 Issue 1, p81 
[4] Bjelica, Duško; Popović, Stevo; Petković, Jovica, Comparison of Instep Kicking Between Preferred and Non-Preferred Leg in Young Football Players. Montenegrin Journal of Sports Science \& Medicine Mar 2013, Vol. 2 Issue 1, p5

[5] Marques, Mário C.; Pereira, Filipe; Marinho, Daniel A.; Reis, Mafalda; Cretu, Marian; Van Den Tillaar, Roland, A comparison of ball velocity in different kicking positions with dominant and non-dominant leg in junior soccer players. Journal of Physical Education \& Sport 2011, Vol. 11 Issue 2, p159

[6] Dörge, H.C.; Andersen, T. Bull; Sørensen, H.; Simonsen, E.B., Biomechanical differences in soccer kicking with the preferred and the non-preferred leg. Journal of Sports Sciences Apr2002, Vol. 20 Issue 4, p293

[7] Cerrah, Ali Onur; Gungor, Elvin Onarıcı; Soylu, Abdullah Ruhi; Ertan, Hayri; Lees, Adrian; Bayrak, Coşkun, Muscular activation patterns during the soccer in-step kick. Isokinetics \& Exercise Science 2011, Vol. 19 Issue 3, p181

[8] Scurr, Joannac.; Abbott, Victoria; Ball, Nick, Quadriceps EMG muscle activation during accurate soccer instep kicking. Journal of Sports Sciences Feb 2011, Vol. 29 Issue 3, p247

[9] Scurr, Joanna; Hall, Ben, The effects of approach angle on penalty kicking accuracy and kick kinematics with recreational soccer players. Journal of Sports Science \& Medicine Jun2009, Vol. 8 Issue 2, p230 